\title{
Sliding Window for Radial Basis Function Neural Network Face Detection
}

\author{
K.A.A Aziz \\ Faculty of Engineering \\ Technology \\ Universiti Teknikal Malaysia \\ Melaka \\ Melaka, Malaysia
}

\author{
N. Mohamood \\ Faculty of Engineering \\ Technology \\ Universiti Teknikal Malaysia \\ Melaka \\ Melaka, Malaysia
}

\author{
M.N.Z. Hashim \\ Faculty of Electronics and \\ Computer Engineering \\ Universiti Teknikal Malaysia \\ Melaka \\ Melaka, Malaysia
}

\begin{abstract}
This paper present a Radial Basis Function Neural Network (RBFNN) face detection using sliding windows. The system will detect faces in a large image where sliding window will run inside the image and identified whether there is a face inside the current window. Face detection is the first step in face recognition system. The purpose is to localize and extract the face region from the background that will be fed into the face recognition system for identification. General preprocessing approach was used for normalizing the image and a Radial Basis Function (RBF) Neural Network was used to distinguish between face and non-face images. RBFNN offer several advantages compared to other neural network architecture such as they can be trained using fast two stages training algorithm and the network possesses the property of best approximation. The output of the network can be optimized by setting suitable values of the center and spread of the RBF. In this paper, a uniform fixed spread value will be used. The performance of the system will be based on the rate of detection and also false negative rate.
\end{abstract}

Keywords: Face detection, Radial Basis Function Neural Network, Sliding Window.

\section{INTRODUCTION}

Biometrics deals with the identification of individuals based on their biological or behavioral characteristics [1]. By using that trait to obtain a biometric enrollment, we can say that with a degree of certainty that someone is the same person in future biometric authentications based on their previous enrollment authentications [2]. A number of biometrics have been proposed, researched and evaluated for identification applications. Face is one of the most acceptable biometrics because it is one of the most common methods of identification which humans use in their interactions [1]. Face detection is the first step in face recognition system. Face detection can be regarded as a more general case of face localization. In face localization, the task is to find the locations and sizes of a known number of faces. One of the methods for face detection is Neural Networks which lies under the category of image based approach. In this paper, we focus on optimizing the RBF Neural Network for face detection. RBFNN is used to distinguish face and non-face images. The output of the network can be optimized by setting suitable values of the center and the spread of the RBF. The system will detect many faces in a large image where sliding window will run inside the image and identified whether there is a face inside the current window.

\section{RADIAL BASIS FUNCTION NEURAL NETWORK}

RBFNN offers several advantages compared to the Multilayer

Perceptrons. Two of these advantages are:

1. They can be trained using fast 2 stages training algorithm without the need for time consuming non-linear optimization techniques.

2. ANN RBF possesses the property of 'best approximation' [9]. This means that if in the set A of approximating functions (for instance the set $\mathrm{F}(\mathrm{x}, \mathrm{w}$ ) spanned by parameters $\mathrm{w}$ ), then the RBFNN has the minimum distance from any given function of a larger set, $\mathrm{H}$.

RBFNN had been successfully used in face detection such as in Mikami, et. al., 2003[3]. Figure 1 illustrates the architecture of the RBFNN used in this work.

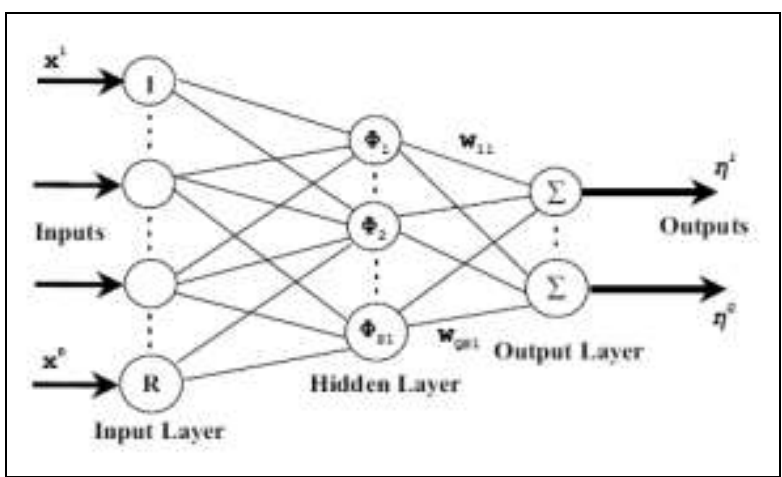

Figure 1: RBF Neural Network

The network consists of three layers: an input layer, a hidden layer and an output layer. Here, $\mathrm{R}$ denotes the number of inputs while $\mathrm{Q}$ the number of outputs. For $\mathrm{Q}=1$, the output of the RBFNN in Figure 1 is calculated according to

$$
\eta(x, w)=\sum_{k=1}^{S 1} w_{1 k} \phi\left(\left\|x-c_{k}\right\|\right)_{2}
$$

where $x \in \mathfrak{R}^{R x 1}$ is an input vector, $\phi($.$) is a basis function,$ $\|$.$\| denotes the Euclidean norm, w1k are the weights in the$ output layer, $\mathrm{S} 1$ is the number of neurons ( and centers) in the 
hidden layer and $c_{k} \in \mathfrak{R}^{R x 1}$ are the RBF centers in the input vector space. Equation (1) can also be written as

$$
\eta(x, w)=\phi^{T}(x) w
$$

where

$$
\phi^{T}(x)=\left[\phi\left(\left\|x-c_{1}\right\|\right) \ldots \ldots \phi_{S 1}\left(\left\|x-c_{S 1}\right\|\right)\right]
$$

and

$$
w^{T}=\left[w_{11} w_{12} \ldots w_{1 S 1}\right]
$$

The output of the neuron in a hidden layer is a nonlinear function of the distance given by:

$$
\phi(x)=e^{\frac{-x^{2}}{\beta^{2}}}
$$

where $\beta$ is the spread parameter of the RBF. For training, the least squares formula was used to find the second layer weights while the centers are set using the available data samples.

\section{NETWORK TRAINING}

The image that to be fed into the network whether for training or testing will be normalized using a preprocessing step, adapted from [4].

In this project, image is first converted into double class in matrix form. The matrix is the converted into column matrix 1 $\mathrm{x} \mathrm{n}$. This input will be fed into the RBF network for the next process.

The network is trained using 2429 face data and 4548 nonface data from the CBCL (Center For Biological and Computation Learning) train datasets [5].

$\mathrm{K}$-means clustering is one of the techniques that was used to find a set of centers where the technique is more accurately reflects the distribution of the data points [6]. It is used in research such as in [3] and [7]. In k-means clustering, the number of desired centers, $\mathrm{K}$, must be decided in advance.

The simplest procedure for selecting the basis function centers $\mathrm{ck}$ is to set the center equal to the input vectors or a random subset of the input vectors from the training set but this is not an optimal procedure since it leads to the use of unnecessarily large number of basis function [6]. Broomhead et al. [8] suggested strategies for selecting RBF centers randomly from the training data. The centers of RBF can either be distributed uniformly within the region of input space for which there is data.

For the training, supervised learning is used where training patterns are provided to the RBFNN together with a teaching signal or target. As for the input of face will be given the value of 1 while the input of non-face will be given the value of -1 .

\section{SLIDING WINDOWS}

Sliding window can be described as a matrix of pixels $(i \times j)$ that moves through the entire pixels of an image. In this project, both the value of $x$ and $y$ are 19 . The matrix is smaller compared to the matrix of the image. Therefore, the matrix can be compared to many parts in the matrix of the image The sliding window has a same size with the distributionbased model matrix. It is matched to the distribution-based model for looking the difference feature vectors. In this project, a window by the size of $19 \times 19$ will be used. The program will first calculate the size of the large image. Then the window will moves through the entire pixels of an image.

For the image with the size of $(\mathrm{x} \mathrm{x} y)$ the sliding window can be given by:

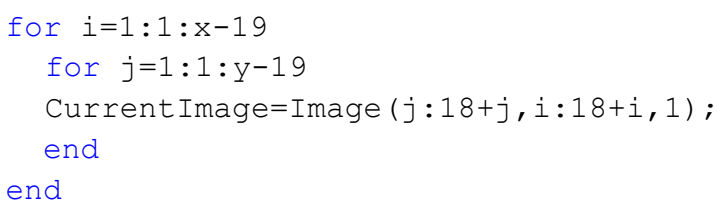

Most of the image-based approaches apply a window scanning technique for detecting faces. The window scanning algorithm is in essence just an exhaustive search of the input image for possible face locations at all scales, but there are variations in the implementation of this algorithm for almost all the image-based systems. Typically, the size of the scanning window, the sub sampling rate, the step size, and the number of iterations vary depending on the method proposed and the need for a computationally efficient system. [9]

\section{TESTING}

The system will detect many faces in a large image. The image for testing is taken from [10]. Sliding window will run inside the image and identified whether there is a face inside the current window. If a face is detected, the system will put a rectangular mark on the face. In [10] they are using new reduced set method for Support Vector Machines (SVMs).

\section{RESULTS}

For detecting many faces in an image, setting the system with center 2 as shown in Figure 2 gives the worst result as there are too many false accept. It is hard to see whether the system detect all faces as there are too many overlapped rectangular. Increasing the value of center improved the result as the false negative becomes less as in Figure 3. For the use of center 5 and spread 10, the system cannot detect two faces and yet have two false accept.

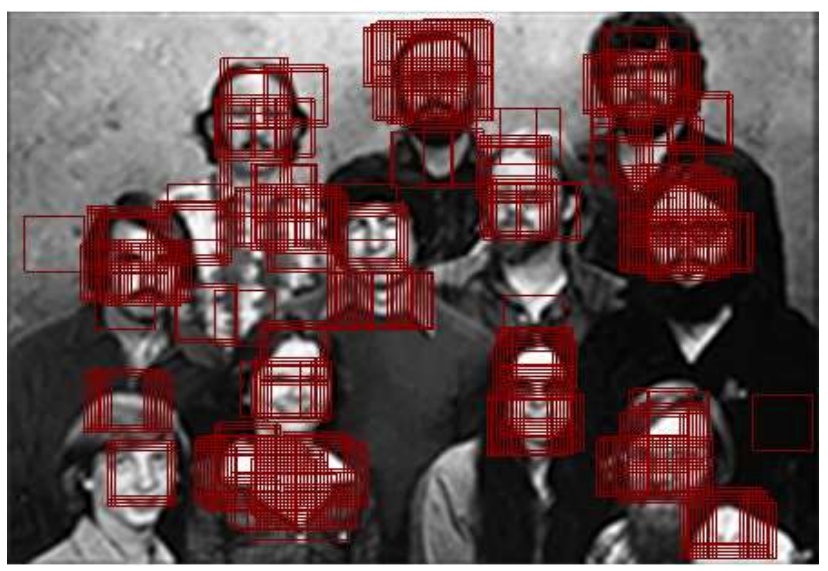

Figure 2: Using RBF with the value of Centre 2 and Spread 4 


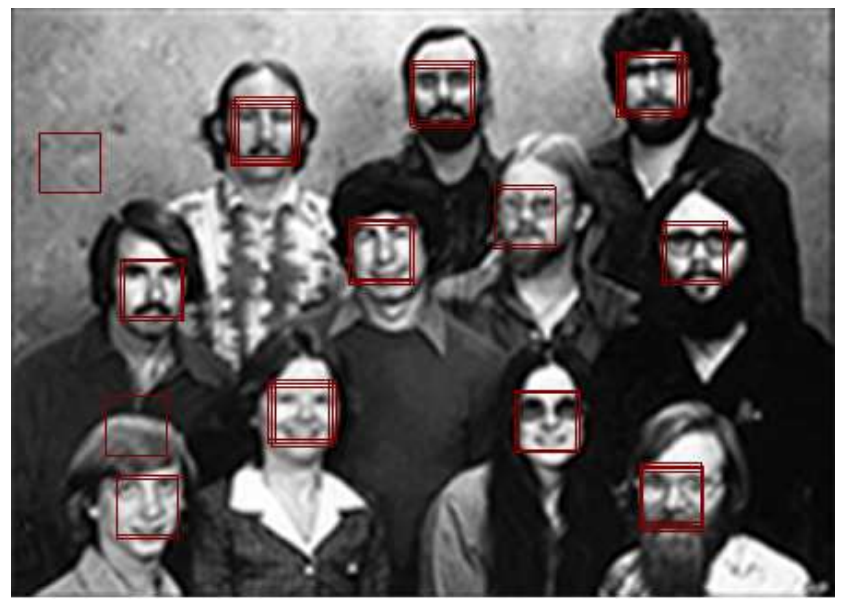

Figure 3: Using RBF with the value of Centre 5 and Spread 4

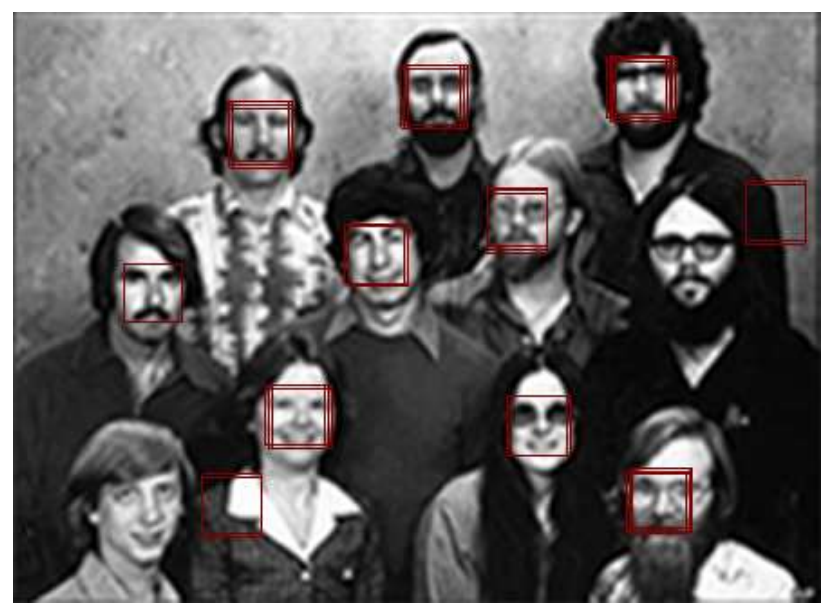

Figure 4: Using RBF with the value of Centre 5 and Spread 10

Starting with center 25 and above, the results are much better as the system can detect all faces in the picture without any false accept as we refer to Figure 5. But still the spread value is crucial where we can still get false accept as shown in Figure 6 and Figure 7 for center 25 and 200.

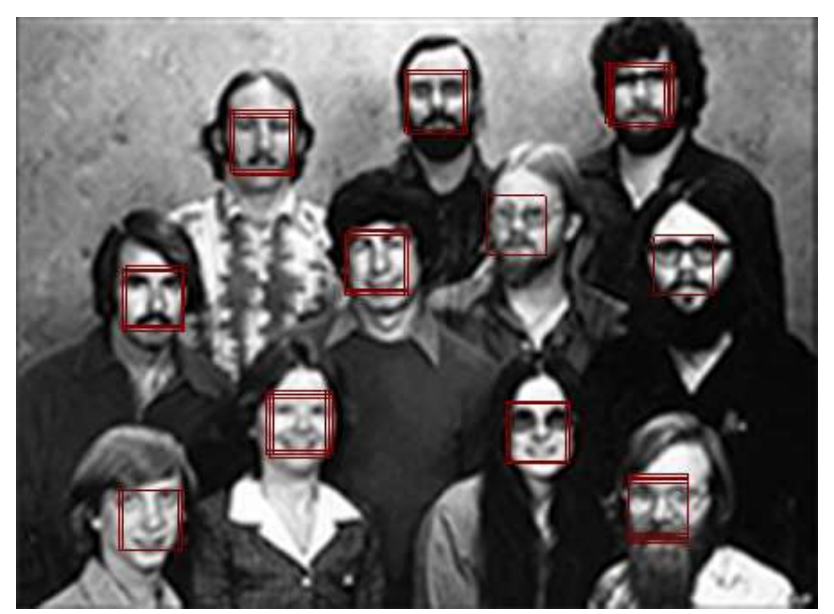

Figure 5: Using RBF with the value of Centre 25 and Spread 5

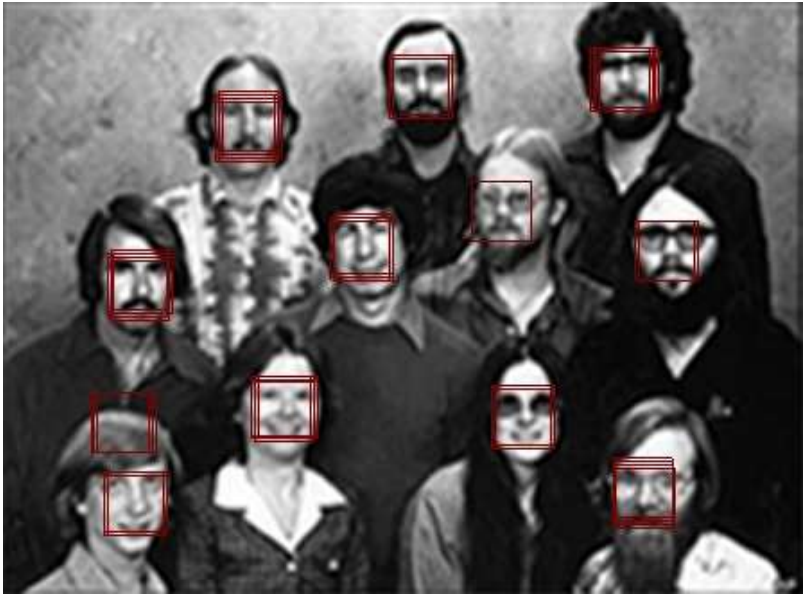

Figure 6: Using RBF with the value of Centre 25 and Spread 4

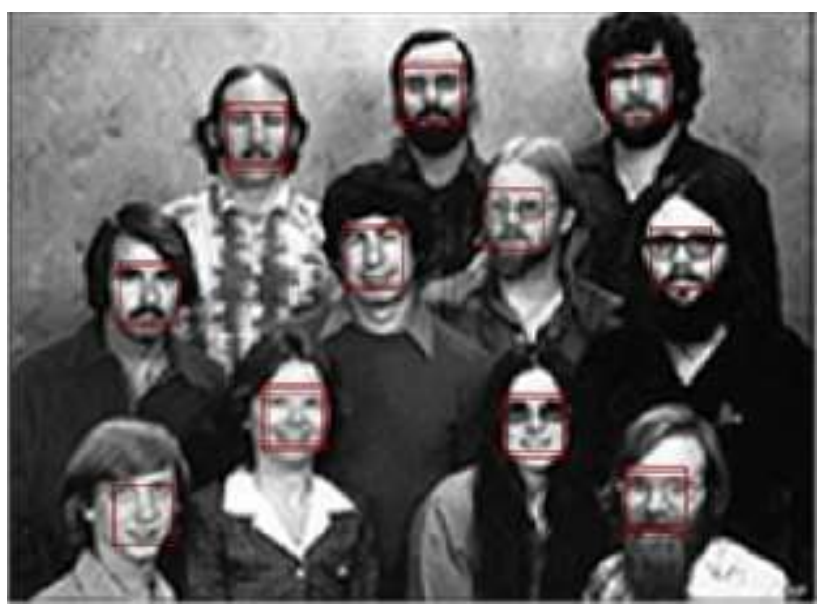

Figure 7: Using RBF with the value of Centre 200 and Spread 5

For Center 200, spread value of 5 will ensure that the system detect all faces in the image without false accept as shown in Figure 7. As for spread 4, the system can detect all faces but with 2 false accept.

\section{CONCLUSION}

In section 5 , setting the center higher than 25 will ensure that all faces in the image will be detected. But still the value of spread is crucial. This can be seen in Figure 6 where there is one false accept. For 25 centers, spread equal to 4 is the best setting. But for center 200, using spread equal to 5 will ensure that the system detects all faces in the image. 


\section{ACKNOWLEDGMENTS}

The authors would like to express their gratitude to the Universiti Teknikal Malaysia Melaka for providing the facilities and financial assistance to conduct this research.

\section{REFERENCES}

[1] A. Jain, R. Bolle, and S. Pankanti, "Biometrics: Personal Identifucation in Networked Society", Springer Science+Business Media, Inc 2006.

[2] P. Reid, "Biometrics for Network Security", Pearson Education, Inc 2004.

[3] T. Mikami, M. Wada, "Example-based Face Detection Using Independent Component Analysis and RBF Network", SICE Annual Conference in Fukui, August 46, 2003.

[4] H. A. Rowley, S. Baluja, and T. Kanade. "Neural Network-Based Face Detection", IEEE Trans. Pattern Analysis and Machine Intelligence, vol. 20, no. 1, pp. 2338. 1998.

[5] CBCL Face Database \#1 MIT Center For Biological and Computation Learning http://www.ai.mit.edu/projects/cbcl

[6] S. S. Abdullah, M. M. Idris, "A Short Course In Artificial Neural Networks" 2008.

[7] M. J. Er, S. Wu, J. Lu, and H. L. Toh, Face Recognition With Radial Basis Function (RBF) Neural Networks, IEEE Transaction On Neural Networks, Vol. 13, No. 3, May 2002.

[8] D. S. Broomhead and D. Lowe, Multivariable functional interpolation and adaptive networks, Complex Systems, vol. 2, pp 321-355.

[9] E. Hjelmas, and B. K. Low, "Face Detection: A Survey" Academic Press, 2001

[10] W. Kienzle, G. Bakir, M. Franz and B. Scholkopf: Face Detection - Efficient and Rank Deficient. In: Advances in Neural Information Processing Systems 17, pg. 673680, 2005.

[11] K.A.A. Aziz, S.S. Abdullah, R.A. Ramlee and A.N. Jahari. "Face Detection Using Radial Basis Function Neural Networks With Variance Spread Value", The International Conference of Soft Computing and Pattern Recognition (SoCPaR 2009) Malacca, Malaysia, December 4-7, 2009.

[12] K.A.A. Aziz, R.A. Hamzah, S.D.I Damni, A.N.M. Johari and S.S. Abdullah. "Face Recognition Using Fixed Spread Radial Basis Function Neural Network For Security System,", Journal of Telecommunication, Electronic And Computer Engineering, 2011.

[13] K.A.A. Aziz, R.A. Ramlee, S.I Samsudin and A.N.M. Johari. "The Effect Of Overlapping Spread Value For Radial Basis Function Neural Network In Face Detection", Journal of Telecommunication, Electronic And Computer Engineering, 2010.

[14] R.A Hamzah, S.F.A. Ghani, A. Din and K.A.A. Aziz. "Visualization of image distortion on camera calibration for stereo vision application", Control System, Computing and Engineering (ICCSCE), 2012.

[15] R.A. Ramlee, K. Azha and R.S.S. Singh. "Detecting Cholesterol Presence with Iris Recognition Algorithm", Universiti Teknikal Malaysia Melaka (UTeM), Malaysia.
[16] R.A. Hamzah, S.F. Abd Ghani, A.F. Kadmin and K.A.A. Aziz. "A practical method for camera calibration in stereo vision mobile robot navigation", Research and Development (SCOReD), 2012.

[17] R.A. Hamzah, K.A.A. Aziz, A.S.M. Shokri. “A pixel to pixel correspondence and region of interest in stereo vision application", Computers \& Informatics (ISCI), 2012.

[18] K.A.A. Aziz, S.S. Abdullah and A.N.M. Johari. "Face Detection Using Radial Basis Functions Neural Networks With Fixed Spread", International Journal of Computer Sciences and Engineering Systems, 2011. 